\title{
The Influence of Critical Boundary Conditions on Finite Difference Solutions of Two Point Boundary Value Problems
}

\author{
By A. R. Mitchell
}

1. Introduction. It is often assumed in the solution of two point boundary problems by finite difference methods that an accurate answer is obtained provided the mesh length is small and the numerical calculations are carried out with sufficient accuracy. The object of the present note is to illustrate by means of worked out examples the extent of the disagreement which often exists, even for small mesh lengths, between corresponding exact solutions of ordinary differential and difference equations. Although the differential equation chosen for purposes of illustration in the present paper is a particularly simple one, the difficulties outlined are quite general and arise in the numerical solution of more complicated equations subject to two point boundary conditions.

The equation considered is

$$
\frac{d^{2} y}{d x^{2}}+p \sqrt{k} \frac{d y}{d x}+k y=0
$$

with the associated second order central difference replacement

$$
\left(1+\frac{1}{2} p h\right) y_{r+1}-\left(2-h^{2}\right) y_{r}+\left(1-\frac{1}{2} p h\right) y_{r-1}=0,
$$

where $p, k$ are constants, $y_{r+1}, y_{r}, y_{r-1}$ are the values of $y$ at $x=(r+1) H, r H$, $(r-1) H$ respectively, and $h=\sqrt{k} H$ (where $H$ is the mesh length). In all cases the range of the problem is from $x=0$ to $x=L$ where

$$
L=(N+1) H,
$$

and $N$ is the number of internal nodes. Corresponding exact solutions of (1) and (2) are compared for two types of boundary condition.

2. Function given at both ends of the range. Consider the boundary conditions $y=0$ at $x=0$ and $y=Y$ at $x=L$. The solution of (1) becomes

$$
y=\frac{\sin \frac{1}{2} \sqrt{4-p^{2}} \sqrt{k} x}{\sin \frac{1}{2} \sqrt{4-p^{2}} l} e^{\frac{1}{2} p(l-\sqrt{k} x)} Y
$$

if $p^{2}<4$, and

$$
y=\frac{\sinh \frac{1}{2} \sqrt{p^{2}-4} \sqrt{k} x}{\sinh \frac{1}{2} \sqrt{p^{2}-4} l} e^{\frac{1}{p(p(l-\sqrt{k} x)} Y}
$$

if $p^{2}>4$, where $l=\sqrt{k} L$. No finite solution exists for general $x$ when

$$
l=\frac{2 r \pi}{\sqrt{4-p^{2}}} . \quad(r=1,2,3, \cdots)
$$

Received September 29, 1958. 
The solution of (2) becomes

$$
y_{r}=\left(\frac{2+p h}{2-p h}\right)^{\frac{N+1-r}{2}} \frac{\sin r \theta}{\sin (N+1) \theta} Y
$$

where

$$
\cos \theta=\frac{2-h^{2}}{\sqrt{4-p^{2} h^{2}}}
$$

if $p^{2}+h^{2}<t$, and

$$
y_{r}=\frac{\lambda^{r}-\mu^{r}}{\lambda^{N+1}-\mu^{N+1}} Y \quad(r=1,2, \cdots N)
$$

where

$$
\lambda, \mu=\frac{2-h^{2} \pm h \sqrt{p^{2}+h^{2}-4}}{2+p h}
$$

if $p^{2}+h^{2}>4$. No finite solution exists at general nodes for ranges satisfying

$$
l=\sqrt{2}(N+1)\left[1-\sqrt{1-\frac{p^{2} l^{2}}{4(N+1)^{2}}} \cos \frac{K \pi}{N+1}\right]^{\frac{1}{2}} \cdot \quad(K=1,2, \cdots N)
$$

Ranges for which either the differential or the difference equation has no finite solution are said to be critical.

It can be shown that for a prescribed value of $K$, a critical range of the difference equation given by (7) either approaches a critical range of the differential equation given by (5) or tends to infinity as $N$ tends to infinity. Due to the presence of the mesh length, however small, in a difference calculation, there is never exact correspondence between critical ranges of differential and difference equations, and an attempt is now made to determine the effect of this lack of correspondence in critical range on the agreement between exact solutions of differential and associated difference equations.

Calculations are carried out for $p=0, \sqrt{2}, 1.9,2.0, \sqrt{4.99}$ and for $h=0.1$, 0.2 . Values of $y$ are obtained from (4) at $x=L / 2$, and from (6) at the node $r=(N+1) / 2$. The difference between corresponding exact solutions of the differential and difference equations. is expressed as a percentage of the solution of the differential equation, and the resulting values are quoted in Table 1. It should be pointed out that when $x=L / 2$, (5) requires modification, and in fact no finite solution of the differential equation exists for

$$
l=\frac{2 r \pi}{\sqrt{4-p^{2}}} \cdot \quad(r=1,3,5, \cdots)
$$

Also the sign in front of the percentage differences is plus or minus depending on whether the difference equation solution is greater or less than the differential equation solution.

There are the following points to be noted in the table:

(1) The values of $l$ for which the differential equation has no finite solution are indicated, and it is clear that poor agreement exists between the exact solutions of the differential and difference equations in the vicinity of these critical values. 
A. R. MITCHELL

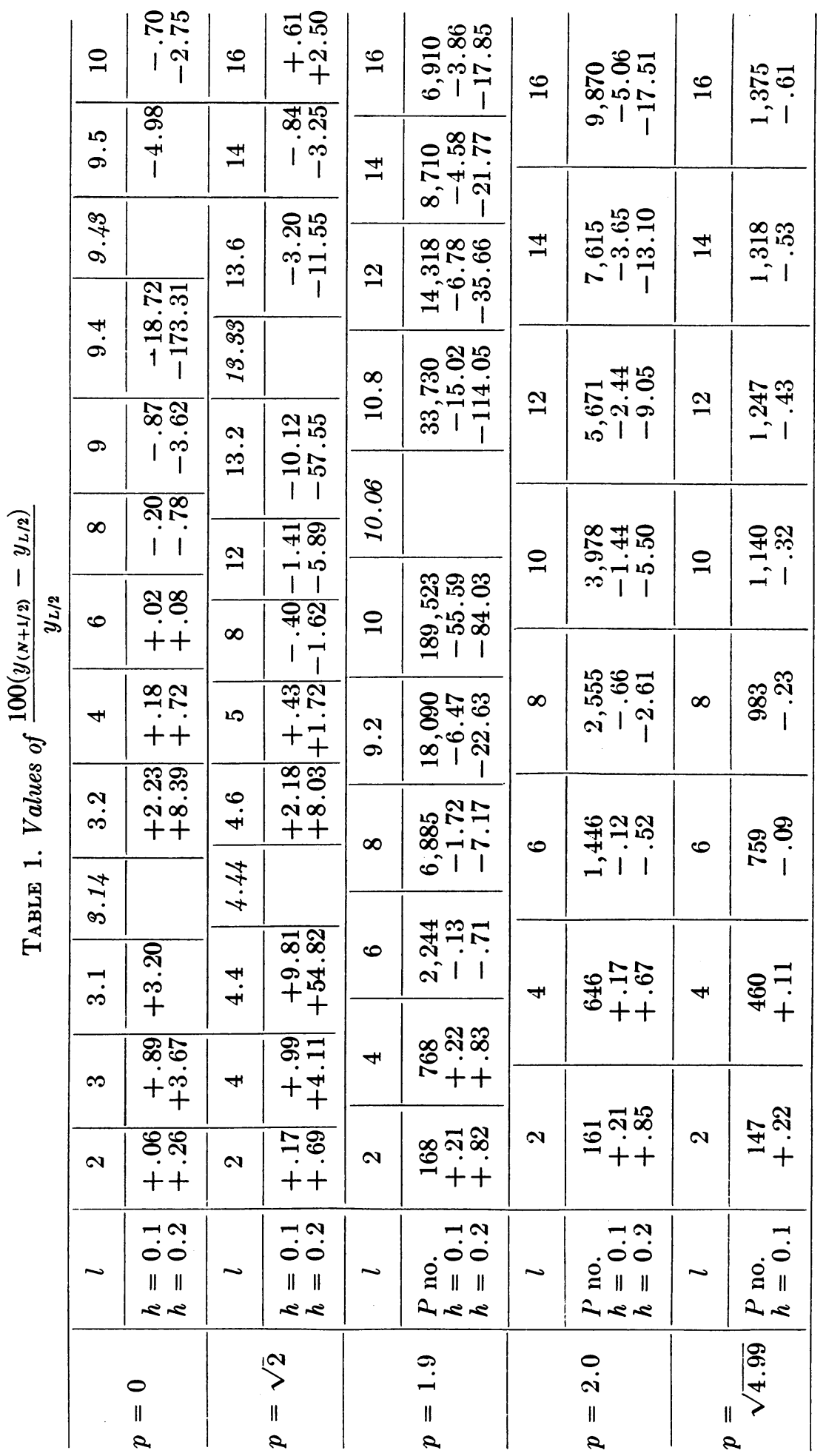


(2) As $p$ approaches the value 2 from below, agreement between the exact solutions deteriorates and when $p=1.9$, for example, the error is in excess of $2 \%$ when $h=0.1$ for any $l>8.2$.

(3) Once $p$ has attained the value 2 , the critical ranges of the differential equation are at infinity and the percentage errors increase monotonically with $l$.

(4) The $P$-condition numbers are given for $p=1.9,2.0, \sqrt{4.99}$ when $h=0.1$. The magnitude of the $P$ number indicates the order of difficulty of obtaining an accurate numerical solution of the appropriate difference equation. The numbers are large in the vicinity of critical ranges.

3. Function and derivative given, one at either end of the range. Next consider the boundary conditions $y=0$ at $x=0$ and $y^{\prime}+\alpha y=\beta$ at $x=L$. The solution of (1) becomes at $x=L$,

$$
y \sqrt{k}=\frac{2 \beta}{\delta-p+\sqrt{4-p^{2}} \cot \sqrt{4-p^{2}} \frac{1}{2} l}
$$

if $p^{2}<4$, and

$$
y \sqrt{k}=\frac{2 \beta}{\delta-p+\sqrt{p^{2}-4} \operatorname{coth} \sqrt{p^{2}-4} \frac{1}{2} l}
$$

if $p^{2}>4$, where $l=\sqrt{k} L$ and $\delta=2 \alpha / \sqrt{k}$. The solution ceases to be finite when

$$
l=\frac{2(r \pi-\phi)}{\sqrt{4-p^{2}}} \quad(r=1,2, \cdots)
$$

where

$$
\phi=\tan ^{-1} \frac{\sqrt{4-p^{2}}}{\delta-p}
$$

if $p^{2}<4$, and when

$$
l=\frac{1}{\sqrt{p^{2}-4}} \log _{e} \frac{\delta-p-\sqrt{p^{2}-4}}{\delta-p+\sqrt{p^{2}+4}}
$$

if $p^{2}>4$. It should be noted that the derivative condition at $x=L$ causes critical ranges to exist for $p^{2}>4$ as well as for $p^{2}<4$.

If the derivative condition at $x=L$ is replaced by

$$
2 y_{N}-\left[\left(2-h^{2}\right)+(2+p h) \frac{1}{2} \delta h\right] y_{N+1}+(2+p h) h \frac{\beta}{\sqrt{k}}=0,
$$

the solution of (2) becomes at the node $r=N+1$,

$$
y \sqrt{k}=-\frac{\beta h(2+p h)}{\left[h^{2}-(2+p h) \frac{1}{2} \delta h-2\right]+2\left(\frac{2+p h}{2-p h}\right)^{\frac{1}{2}} \frac{\sin N \theta}{\sin (N+1) \theta}}
$$


where

$$
\cos 2 \theta=\frac{1+\left(\frac{1}{4} p^{2}-2\right) h^{2}+\frac{1}{2} h^{4}}{1-\frac{1}{4} p^{2} h^{2}}
$$

if $p^{2}+h^{2}<4$, and

$$
y \sqrt{k}=-\frac{\beta h(2+p h)}{\left[h^{2}-(2+p h) \frac{1}{2} \delta h-2\right]+2 \frac{\lambda^{N}-\mu^{N}}{\lambda^{N+1}-\mu^{N+1}}}
$$

where

$$
\lambda, \mu=\frac{2-h^{2} \pm h \sqrt{p^{2}+h^{2}-4}}{2+p h}
$$

if $p^{2}+h^{2}>4$. No finite solution exists when

$$
l=2(N+1)\left[1-\sqrt{1-\frac{p^{2} l^{2}}{4(N+1)^{2}}} \cos \frac{K \pi-\phi}{N+1}\right]^{\frac{1}{2}} \quad(K=1,2, \cdots N)
$$

where

$$
\tan \phi=\frac{\left[\left(4-h^{2}\right)-p^{2}\right]^{\frac{1}{2}}}{\delta\left(1-\frac{1}{4} p^{2} h^{2}\right)+\frac{1}{2} p\left(h^{2}-2\right)}
$$

if $p^{2}+h^{2}<4$, and when

$$
\begin{aligned}
& {\left[\frac{h^{2}-h \sqrt{p^{2}+h^{2}-4}-2}{h^{2}+h \sqrt{p^{2}+h^{2}-4}-2}\right]^{N+1}} \\
& \quad=\frac{\delta\left(1-\frac{1}{4} p^{2} h^{2}\right) h+\frac{1}{2} p\left(h^{2}-2\right) h-\sqrt{p^{2}+h^{2}-4}}{\delta\left(1-\frac{1}{4} p^{2} h^{2}\right) h+\frac{1}{2} p\left(h^{2}-2\right) h+\sqrt{p^{2}+h^{2}-4}}
\end{aligned}
$$

if $p^{2}+h^{2}>4$. Again, it can be shown that the critical ranges of the difference equation given by (11) approach the corresponding critical ranges of the differential equation given by (9) or tend to infinity as $N$ tends to infinity.

Calculations are carried out for $p=1.9,2,3$ with $h=0.1$. A range of values of $\delta$ is covered for each value of $p$. The values of $y \sqrt{k} / \beta$ for the differential and difference equations are obtained from (8a) and (10a) respectively when $p=1.9$, and from $(8 \mathrm{~b})$ and $(10 \mathrm{~b})$ respectively when $p=2$, 3 . In Table 2 , the percentage differences between corresponding exact solutions of the differential and difference equations are quoted for $p=2,3$, whereas the actual differences between corresponding exact solutions are quoted in units of .0001 for $p=1.9$. This is because the exact solution of the differential equation for $p=1.9$ is sometimes so small that the percentage differences are misleadingly high.

There are several points arising from Table 2.

(1) An entry $\infty$ in the table means that the differential equation has no finite solution for the appropriate values of $l$ and $\delta$. As in Table 1, poor agreement exists between exact solutions in the vicinity of these critical values.

(2) When $p=1.9$, critical values occur periodically for each value of $\delta$, but when $p=2,3$ there is at most one critical range for each value of $\delta$. For example 


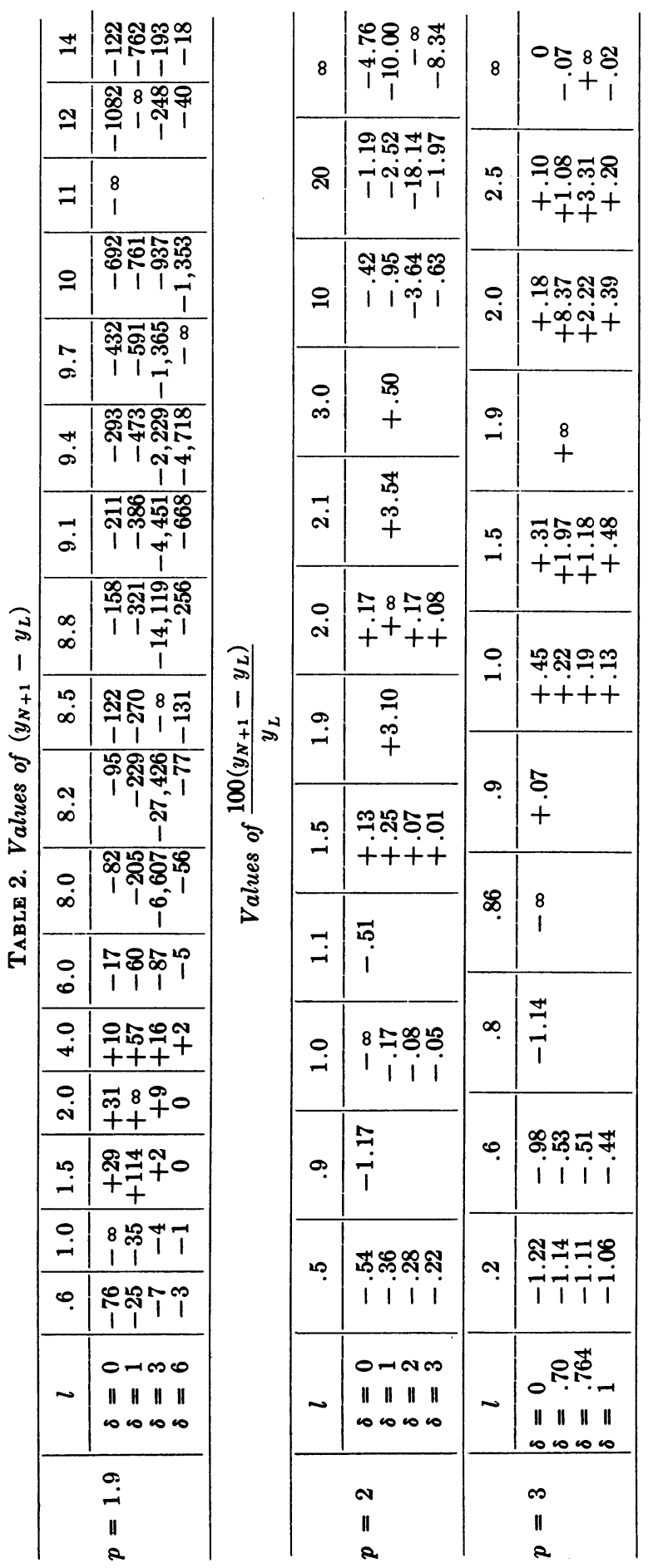


when $p=2$, as $\delta$ increases from 0 to 2 , the value of the critical range increases from 1 to $\infty$. For any value of $\delta>2$, there is no critical range.

(3) The lack of agreement between exact solutions in the vicinity of critical ranges seems to be less pronounced than was the case in Table 1 .

4. The effect of reducing the mesh length on the accuracy of finite difference solutions. So far, exact solutions of the difference equation have been obtained for mesh lengths of 0.1 or 0.2 . Calculations are now carried out to show the effect of reducing the mesh length on the accuracy of finite difference solutions.

Considered the differential equation

$$
\frac{d^{2} y}{d x^{2}}+p \sqrt{k} \frac{d y}{d x}-k y=0
$$

subject to the boundary conditions $y=0$ at $x=0$ and $y^{\prime}+\alpha y=\beta$ at $x=L$. The values of $y \sqrt{k} / \beta$ are calculated from the conventional second order central difference replacement for a range of values of $\delta$ and $N$ when $\sqrt{k} L=4$ and $p=-6$, and are shown in Table 3.

The differential equation itself has no finite solution when $\delta=-12.32$, whilst the difference equation has no finite solution at the following values of $\delta$ and $N$

$\begin{array}{ccccccccc}N & 3 & 10 & 12 & 15 & 31 & 63 & 127 & \infty \\ \delta & +1.95 & +66.98 & -85.28 & -28.61 & -14.40 & -12.79 & -12.44 & -12.32 .\end{array}$

It is the lack of correspondence between critical conditions of the differential and difference equations which causes poor agreement to exist between the exact solutions. For example, when $\sqrt{k} L=4, \delta=-15$, the difference solution at $x=L$ is -.1180 for $N=3$, and as $N$ tends to 29 , the solution increases monotonically to $+\infty$. For $N>29$, the solution increases from $-\infty$ reaching the value -.9034 when $N=63$. Thus although the mesh length $h(=\sqrt{k} H)$ is as small as $\frac{1}{16}$, the exact solution of the difference equation is approximately $20 \%$ less than the exact solution of the differential equation. Of course, for values of $\delta$ less than -15 , the infinite step in the solution of the difference equation occurs at a value of $N$ less than 29, (see Table 3) and so the solution of the difference equation for $N=63$ is correspondingly more accurate. When $\delta=-30$, for example, the error is reduced to $2.65 \%$, which nevertheless is still appreciably large.

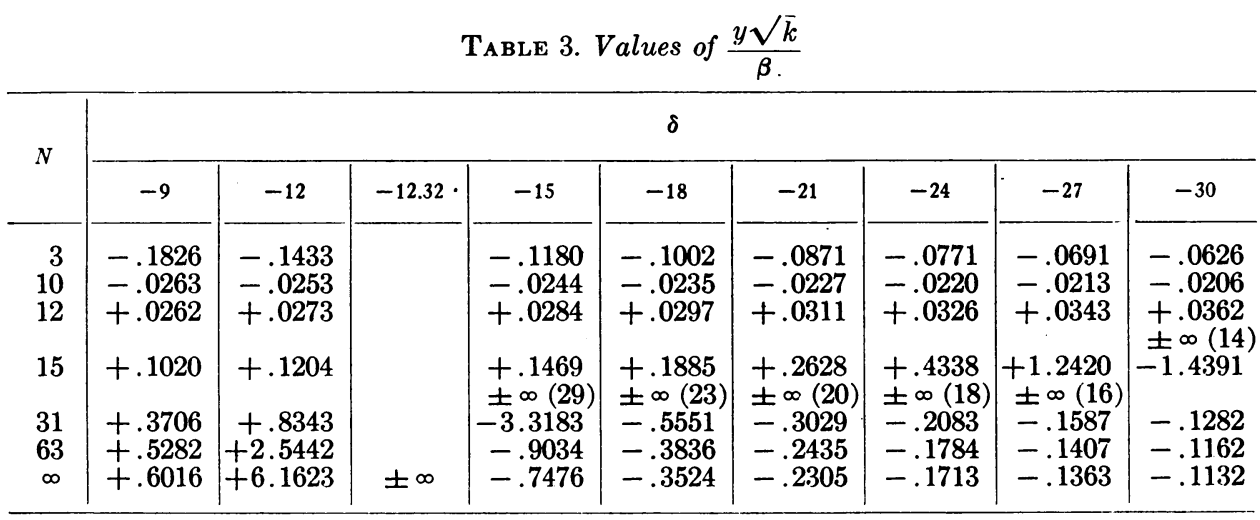




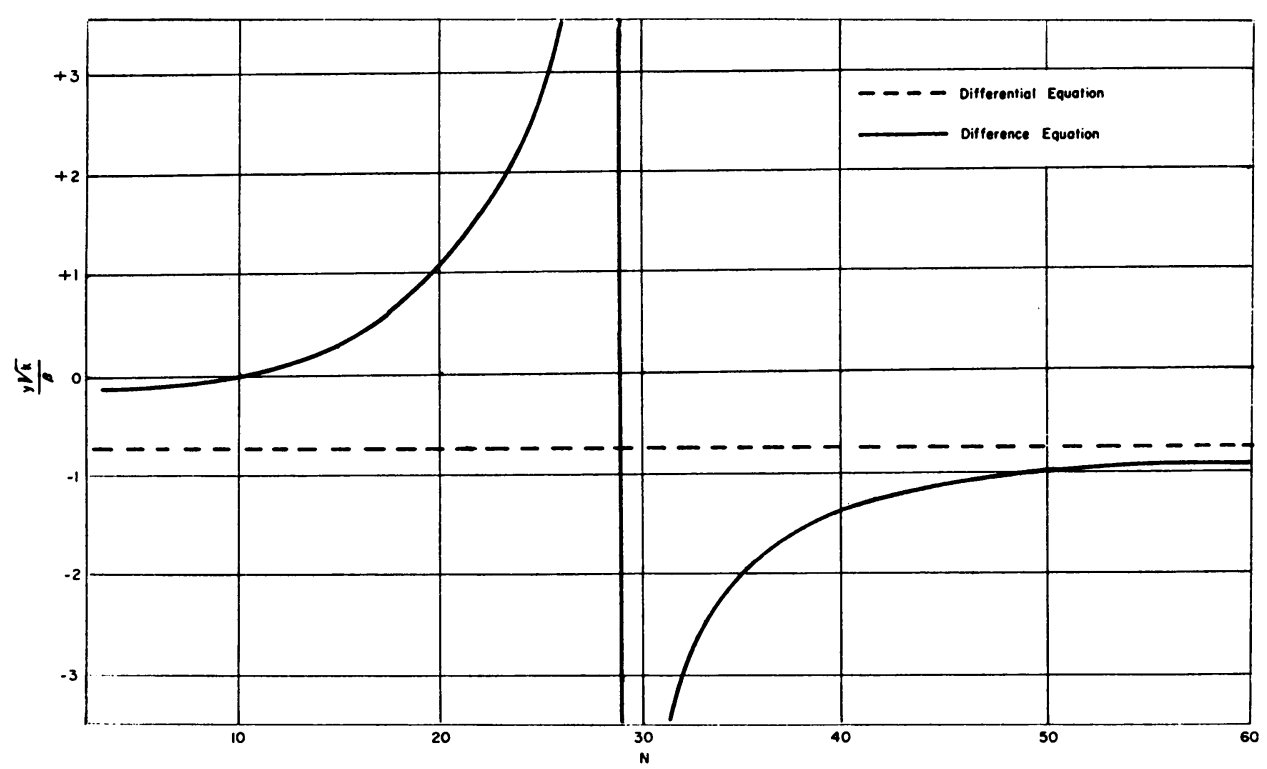

Fig. 1.-Convergence of finite difference solution toward solution of differential equation.

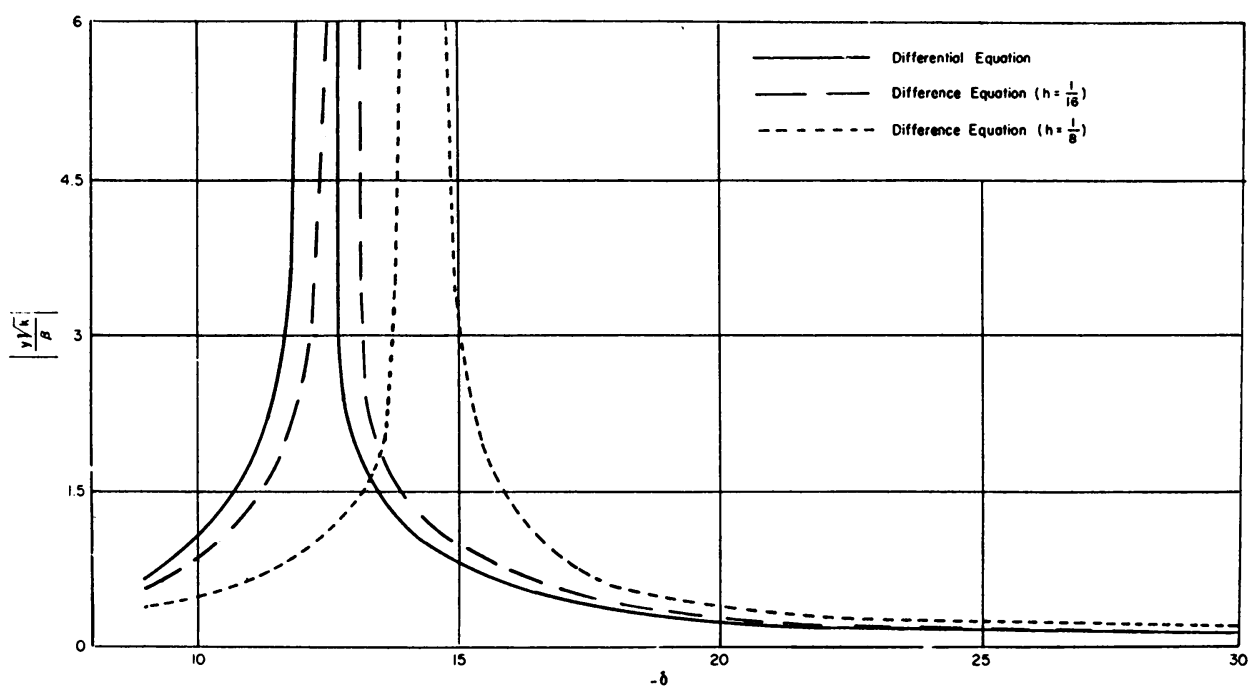

FIG. 2.-Comparison of finite difference solution with solution of differential equation for varying $\delta$.

Two figures are now given to illustrate some of the results contained in Table 3. Fig. 1 illustrates the manner in which the exact solution of the difference equation converges towards the exact solution of the differential equation as the number of internal nodes is increased. The outstanding feature is the infinite step in the solution. Reduction of the mesh length only improves the solution of the difference equation once the infinite step has been passed. Fig. 2 compares the exact solutions 
of the difference equation when $h=\frac{1}{8}, \frac{1}{16}$ respectively with the exact solution of the differential equation, for a range of values of $\delta$. The solutions are infinite at $\delta=-14.40,-12.79$, and -12.32 respectively, and the diagram clearly illustrates how this difference in the critical value of $\delta$ causes poor agreement to exist between the exact solutions over a wide range of $\delta$.

5. Concluding remarks. Although in the present note, no attempt has been made at an exhaustive study of the accuracy of finite difference solutions of two point boundary problems, sufficient examples have been given to show the lack of agreement which can exist between exact solutions of a differential equation and its associated central difference replacement. It is also unlikely that conditions are improved by using alternative difference schemes either of the same or of higher order, since critical conditions of the differential equation have their counterparts in all difference replacements. In fact the use of higher order replacements may well complicate the situation further by introducing additional critical conditions into the system. This was certainly found to be the case in step-by-step difference solutions of initial value problems by Todd [1].

An important point illustrated by the results in Fig. 1 is that in order to guarantee a reasonable answer using difference methods it is necessary but by no means sufficient for the exact solution of the difference equation to tend to the exact solution of the differential equation as the mesh length tends to zero. In fact, the computor is well advised to obtain solutions of the difference equation for a range of mesh lengths in order to detect any infinite step in the solution of the difference equation. For example, in Fig. 1, convergence occurs only for $h<\frac{2}{15}$.

Finally, with regard to the accuracy of the numerical results presented in the present paper, it should be stated that all calculations were carried out on a $10 \times 10$ electric desk machine, the latter being used to its full capacity in all calculations.

\footnotetext{
Department of Mathematics,

St. Andrews University,

Scotland; and

Department of Mathematics,

California Institute of Technical, Pasadena, California.
}

1. J. TopD, "Solution of differential equations by recurrence relations," MTAC, v. 4, 1950 , p. $39-44$. 